\title{
Enumerating Pattern Avoidance for Affine Permutations
}

\author{
Andrew Crites* \\ Department of Mathematics \\ University of Washington, Seattle, WA, USA \\ acrites@math.washington.edu \\ Submitted: Feb 9, 2010; Accepted: July 14, 2010; Published: Sep 22, 2010 \\ Mathematics Subject Classification: 05A05
}

\begin{abstract}
In this paper we study pattern avoidance for affine permutations. In particular, we show that for a given pattern $p$, there are only finitely many affine permutations in $\widetilde{S}_{n}$ that avoid $p$ if and only if $p$ avoids the pattern 321 . We then count the number of affine permutations that avoid a given pattern $p$ for each $p$ in $S_{3}$, as well as give some conjectures for the patterns in $S_{4}$.
\end{abstract}

\section{Introduction}

Given a property $Q$, it is a natural question to ask if there is a simple characterization of all permutations with property $Q$. For example, it is shown in Lakshmibai and Sandhya [1990] that the permutations corresponding to smooth Schubert varieties are exactly the permutations that avoid the two patterns 3412 and 4231. In Tenner [2007] it was shown that the permutations with Boolean order ideals are exactly the ones that avoid the two patterns 321 and 3412. For more examples, a searchable database listing which classes of permutations avoid certain patterns can be found at Tenner [2009]. Since we know pattern avoidance can be used to describe useful sets of permutations, we might ask if we can enumerate the permutations avoiding a given pattern or set of patterns. The goal of this paper is to carry out this enumeration for affine permutations.

We can express elements of the affine symmetric group, $\widetilde{S}_{n}$, as an infinite sequence of integers, and it is still natural to ask if there exists a subsequence with a given relative order. Thus we can extend the notion of pattern avoidance to these affine permutations and we can try to count how many $\omega \in \widetilde{S}_{n}$ avoid a given pattern.

*Andrew Crites acknowledges support from grant DMS-0800978 from the National Science Foundation. 
For $p \in S_{m}$, let

$$
f_{n}^{p}=\#\left\{\omega \in \widetilde{S}_{n}: \omega \text { avoids } p\right\}
$$

and consider the generating function

$$
f^{p}(t)=\sum_{n=2}^{\infty} f_{n}^{p} t^{n}
$$

For a given pattern $p$ there could be infinitely many $\omega \in \widetilde{S}_{n}$ that avoid $p$. In this case, the generating function in (2) is not even defined. As a first step towards understanding $f^{p}(t)$, we will prove the following theorem.

Theorem 1. Let $p \in S_{m}$. For any $n \geqslant 2$ there exist only finitely many $\omega \in \widetilde{S}_{n}$ that avoid $p$ if and only if $p$ avoids the pattern 321.

It is worth noting that 321-avoiding permutations and 321-avoiding affine permutations appear as an interesting class of permutations in their own right. In [Billey et al., 1993, Theorem 2.1] it was shown that a permutation is fully commutative if and only if it is 321-avoiding. This means that every reduced expression for $\omega$ may be obtained from any other reduced expression using only relations of the form $s_{i} s_{j}=s_{j} s_{i}$ with $|i-j|>1$. Moreover, a proof that this result can be extended to affine permutations as well appears in [Green, 2002, Theorem 2.7]. For a detailed discussion of fully commutative elements in other Coxeter groups, see Stembridge [1996].

Even in the case where there might be infinitely many $\omega \in \widetilde{S}_{n}$ that avoid a pattern $p$, we can always construct the following generating function. Let

$$
g_{m, n}^{p}=\#\left\{\omega \in \widetilde{S}_{n}: \omega \text { avoids } p \text { and } \ell(\omega)=m\right\}
$$

Then set

$$
g^{p}(x, y)=\sum_{n=2}^{\infty} \sum_{m=0}^{\infty} g_{m, n}^{p} x^{m} y^{n} .
$$

Since there are only finitely many elements in $\widetilde{S}_{n}$ of a given length, we always have $g_{m, n}^{p}<\infty$. The generating function $g^{321}(x, y)$ is computed in [Hanusa and Jones, 2009, Theorem 3.2].

The outline of this paper is as follows. In Section 2 we will review the definition of the affine symmetric group and list several of its useful properties. In Section 3 we will prove Theorem 1, which will follow immediately from combining Propositions 4 and 5. In Section 4 we will compute $f^{p}(t)$ for all of the patterns in $S_{3}$. Finally, in Section 5 we will give some basic results and conjectures for $f^{p}(t)$ for the patterns in $S_{4}$. 


\section{Background}

Let $\widetilde{S}_{n}$ denote of the set of all bijections $\omega: \mathbb{Z} \rightarrow \mathbb{Z}$ with $\omega(i+n)=\omega(i)+n$ for all $i \in \mathbb{Z}$ and

$$
\sum_{i=1}^{n} \omega(i)=\left(\begin{array}{c}
n+1 \\
2
\end{array}\right) .
$$

$\widetilde{S}_{n}$ is called the affine symmetric group, and the elements of $\widetilde{S}_{n}$ are called affine permutations. This definition of affine permutations first appeared in [Lusztig, 1983, §3.6] and was then developed in Shi [1986]. Note that $\widetilde{S}_{n}$ also occurs as the affine Weyl group of type $\widetilde{A}_{n}$.

We can view an affine permutation in its one-line notation as the infinite string of integers

$$
\cdots \omega_{-1} \omega_{0} \omega_{1} \cdots \omega_{n} \omega_{n+1} \cdots,
$$

where, for simplicity of notation, we write $\omega_{i}=\omega(i)$. An affine permutation is completely determined by its action on $[n]:=\{1, \ldots, n\}$. Thus we only need to record the base window $\left[\omega_{1}, \ldots, \omega_{n}\right]$ to capture all of the information about $\omega$. Sometimes, however, it will be useful to write down a larger section of the one-line notation.

Given $i \not \equiv j \bmod n$, let $t_{i j}$ denote the affine transposition that interchanges $i+m n$ and $j+m n$ for all $m \in \mathbb{Z}$ and leaves all $k$ not congruent to $i$ or $j$ fixed. Since $t_{i j}=t_{i+n, j+n}$ in $\widetilde{S}_{n}$, it suffices to assume $1 \leqslant i \leqslant n$ and $i<j$. Note that if we restrict to the affine permutations with $\left\{\omega_{1}, \ldots, \omega_{n}\right\}=[n]$, then we get a subgroup of $\widetilde{S}_{n}$ isomorphic to $S_{n}$, the group of permutations of $[n]$. Hence if $1 \leqslant i<j \leqslant n$, the above notion of transposition is the same as for the symmetric group.

Given a permutation $p \in S_{k}$ and an affine permutation $\omega \in \widetilde{S}_{n}$, we say that $\omega$ avoids the pattern $p$ if there is no subsequence of integers $i_{1}<\cdots<i_{k}$ such that the subword $\omega_{i_{1}} \cdots \omega_{i_{k}}$ of $\omega$ has the same relative order as the elements of $p$. Otherwise, we say that $\omega$ contains $p$. For example, if $\omega=[8,1,3,5,4,0] \in \widetilde{S}_{6}$, then $8,1,5,0$ is an occurrence of the pattern 4231 in $\omega$. However, $\omega$ avoids the pattern 3412. A pattern can also come from terms outside of the base window $\left[\omega_{1}, \ldots, \omega_{n}\right]$. In the previous example, $\omega$ also has $2,8,6$ as an occurrence of the pattern 132. Choosing a subword $\omega_{i_{1}} \cdots \omega_{i_{k}}$ with the same relative order as $p$ will be referred to as placing $p$ in $\omega$.

\subsection{Coxeter Groups}

For a general reference on the basics of Coxeter groups, see Björner and Brenti [2005] or Humphreys [1990]. Let $S=\left\{s_{1}, \ldots, s_{n}\right\}$ be a finite set, and let $F$ denote the free group on the set $S$. Here the group operation is concatenation of words, so that the empty word is the identity element. Let $M=\left(m_{i j}\right)_{i, j=1}^{n}$ be any symmetric $n \times n$ matrix whose entries come from $\mathbb{Z}_{>0} \cup\{\infty\}$ with 1's on the diagonal and $m_{i j}>1$ if $i \neq j$. Then let $N$ be the normal subgroup of $F$ generated by the relations

$$
R=\left\{\left(s_{i} s_{j}\right)^{m_{i j}}=1\right\}_{i, j=1}^{n} .
$$


If $m_{i j}=\infty$, then there is no relationship between $s_{i}$ and $s_{j}$. The Coxeter group corresponding to $S$ and $M$ is the quotient group $W=F / N$.

Any $w \in W$ can be written as a product of elements from $S$ in infinitely many ways. Every such word will be called an expression for $w$. Any expression of minimal length will be called a reduced expression, and the number of letters in such an expression will be denoted $\ell(w)$, the length of $w$. Call any element of $S$ a simple reflection and any element conjugate to a simple reflection, a reflection.

We graphically encode the relations in a Coxeter group via its Coxeter graph. This is the labeled graph whose vertices are the elements of $S$. We place an edge between two vertices $s_{i}$ and $s_{j}$ if $m_{i j}>2$ and we label the edge $m_{i j}$ whenever $m_{i j}>3$. The Coxeter graphs of all the finite Coxeter groups have been classified. See, for example, [Humphreys, $1990, \S 2]$.

In [Björner and Brenti, 2005, §8.3] it was shown that $\widetilde{S}_{n}$ is the Coxeter group with generating set $S=\left\{s_{0}, s_{1}, \ldots, s_{n-1}\right\}$, and relations

$$
R= \begin{cases}s_{i}^{2}=1, & \\ \left(s_{i} s_{j}\right)^{2}=1, & \text { if }|i-j| \geqslant 2 \\ \left(s_{i} s_{i+1}\right)^{3}=1, & \text { for } 0 \leqslant i \leqslant n-1\end{cases}
$$

where all of the subscripts are taken $\bmod n$. Thus the Coxeter graph for $\widetilde{S}_{n}$ is an $n$-cycle, where every edge is unlabeled.

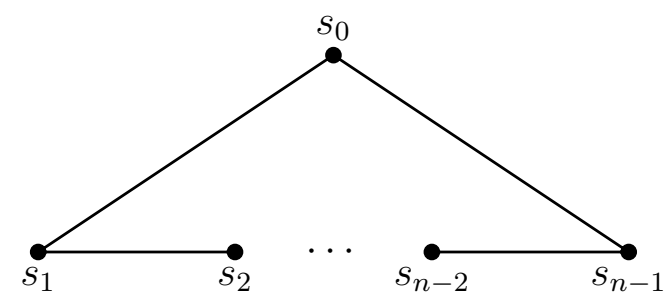

Figure 1: Coxeter graph for $\widetilde{S}_{n}$.

If $J \subsetneq S$ is a proper subset of $S$, then we call the subgroup of $W$ generated by just the elements of $J$ a parabolic subgroup. Denote this subgroup by $W_{J}$. In the case of the affine symmetric group we have the following characterization of parabolic subgroups, which follows easily from the fact that, when $J=S \backslash\left\{s_{i}\right\},\left(\widetilde{S}_{n}\right)_{J}=\operatorname{Stab}([i, i+n-1])$ [Björner and Brenti, 2005, Proposition 8.3.4].

Proposition 2. Let $J=S \backslash\left\{s_{i}\right\}$. Then $\omega \in \widetilde{S}_{n}$ is in the parabolic subgroup $\left(\widetilde{S}_{n}\right)_{J}$ if and only if there exists some integer $i \leqslant j \leqslant i+n-1$ such that $\omega_{j} \leqslant \omega_{k}<\omega_{j}+n$ for all $i \leqslant k \leqslant i+n-1$. 


\subsection{Length Function for $\widetilde{S}_{n}$}

For $\omega \in \widetilde{S}_{n}$, let $\ell(\omega)$ denote the length of $\omega$ when $\widetilde{S}_{n}$ is viewed as a Coxeter group. Recall that for a non-affine permutation $\pi \in S_{n}$ we can define an inversion as a pair $(i, j)$ such that $i<j$ and $\pi_{i}>\pi_{j}$. For an affine permutation, if $\omega_{i}>\omega_{j}$ for some $i<j$, then we also have $\omega_{i+k n}>\omega_{j+k n}$ for all $k \in \mathbb{Z}$. Hence any affine permutation with a single inversion has infinitely many inversions. Thus we standardize each inversion as follows. Define an affine inversion as a pair $(i, j)$ such that $1 \leqslant i \leqslant n, i<j$, and $\omega_{i}>\omega_{j}$. If we let $\operatorname{Inv}_{\widetilde{S}_{n}}(\omega)$ denote the set of all affine inversions in $\omega$, then $\ell(\omega)=\# \operatorname{Inv}_{\widetilde{S}_{n}}(\omega),[$ Björner and Brenti, 2005, Proposition 8.3.1].

We also have the following characterization of the length of an affine permutation, which will be useful later.

Theorem 3. [Shi, 1986, Lemma 4.2.2] Let $\omega \in \widetilde{S}_{n}$. Then

$$
\ell(\omega)=\sum_{1 \leqslant i<j \leqslant n}\left\lfloor\mid \frac{\omega_{j}-\omega_{i}}{n}\right\rfloor \mid=\operatorname{inv}\left(\omega_{1}, \ldots, \omega_{n}\right)+\sum_{1 \leqslant i<j \leqslant n}\left\lfloor\frac{\left|\omega_{j}-\omega_{i}\right|}{n}\right\rfloor,
$$

where $\operatorname{inv}\left(\omega_{1}, \ldots, \omega_{n}\right)=\#\left\{1 \leqslant i<j \leqslant n: \omega_{i}>\omega_{j}\right\}$.

For $1 \leqslant i \leqslant n$ define $\operatorname{Inv}_{i}(\omega)=\#\left\{j \in \mathbb{N}: i<j, \omega_{i}>\omega_{j}\right\}$. Now let $\operatorname{Inv}(\omega)=$ $\left(\operatorname{Inv}_{1}(\omega), \ldots, \operatorname{Inv}_{n}(\omega)\right)$, which will be called the affine inversion table of $\omega$. In [Björner and Brenti, 1996, Theorem 4.6] it was shown that there is a bijection between $\widetilde{S}_{n}$ and elements of $\mathbb{Z}_{\geqslant 0}^{n}$ containing at least one zero entry.

\section{Proof of Theorem 1}

We start with the proof of one direction of Theorem 1. Proposition 5 will complete the proof.

Proposition 4. If $p \in S_{m}$ contains the pattern 321, then there are infinitely many $\omega \in \widetilde{S}_{n}$ that avoid $p$.

Proof. For $k \in \mathbb{N}$, let $\omega^{(k)} \in \widetilde{S}_{n}$ be the affine permutation whose reduced expression, when read right to left, is obtained as follows. Starting at $s_{0}$, proceed clockwise around the Coxeter diagram in Figure $1 k(n-1)$ steps, appending each vertex as you go. The base window of the one-line notation of these elements has the form

$$
\omega^{(k)}=[1-k, 2-k, \ldots, n-1-k, n+k(n-1)] .
$$

Note these elements correspond with the spiral varieties in the affine Grassmannian from Billey and Mitchell [2009].

As an example, in $\widetilde{S}_{4}$ we have the following:

$$
\begin{aligned}
s_{2} s_{1} s_{0} & =\omega^{(1)}=[0,1,2,7] \\
s_{1} s_{0} s_{3} s_{2} s_{1} s_{0} & =\omega^{(2)}=[-1,0,1,10] \\
s_{0} s_{3} s_{2} s_{1} s_{0} s_{3} s_{2} s_{1} s_{0} & =\omega^{(3)}=[-2,-1,0,13] .
\end{aligned}
$$


The infinite string in the one-line notation of $\omega^{(k)}$ is a shuffle of two increasing sequences. Hence every $\omega^{(k)}$ avoids the pattern 321. Thus there are infinitely many permutations in $\widetilde{S}_{n}$ avoiding the pattern 321 , and hence avoiding any pattern $p$ containing 321.

Call a permutation $p \in S_{m}$ decomposable if $p$ is contained in a proper parabolic subgroup of $S_{m}$. Note this is also called sum decomposable by other authors. In other words, there exists some $1 \leqslant j \leqslant m-1$ such that $\left\{p_{1}, \ldots, p_{j}\right\}=\{1, \ldots, j\}$. We also have $\left\{p_{j+1}, \ldots, p_{m}\right\}=\{j+1, \ldots, m\}$, so that we can view $q=p_{1} \cdots p_{j}$ as an element of $S_{j}$ and $r=p_{j+1} \cdots p_{m}$ as an element of $S_{m-j}$. In this case, write $p=q \oplus r$.

Proposition 5. Let $p \in S_{m}$ and $\omega \in \widetilde{S}_{n}$. If $p$ avoids the pattern 321, then there exists some constant $L$ such that if $\ell(\omega)>L$, then $\omega$ contains the pattern $p$. Hence there are only finitely many $\omega \in \widetilde{S}_{n}$ that avoid $p$.

Proof. If $p$ is decomposable, then we can write $p=q_{1} \oplus \cdots \oplus q_{k}$, where each $q_{i}$ is not decomposable. Suppose that for each $1 \leqslant i \leqslant k$, there exists an $L_{i}$ such that, if $\ell(\omega)>L_{i}$, then $\omega$ contains $q_{i}$. Set $L=\max \left\{L_{1}, \ldots, L_{k}\right\}$. If $\ell(\omega)>L$, then $\omega$ contains each of the $q_{i}$. By the periodicity property of $\omega$, we may translate the occurrence of each $q_{i}$ in $\omega$ to the right, so that it lies strictly between the occurrence of $q_{i-1}$ and $q_{i+1}$. Since the values of $q_{i}$ lie between the values of $q_{i-1}$ and $q_{i+1}$, this gives an occurrence of $p$ in $\omega$. Hence, it suffices to assume $p$ is not decomposable.

Let $a=a_{1} \cdots a_{\ell}$ be the subsequence of $p$ consisting of all $p_{j}$ such that $p_{i}<p_{j}$ for all $i<j$. Here $a$ is just the sequence of left-to-right maxima. Let $b$ be the subsequence of $p$ consisting of all $p_{i}$ not in $a$. By its construction, $a$ must be increasing. Furthermore, since $p$ avoids the pattern $321, b$ must also be increasing. To see this, note that if there is some $p_{s}, p_{t}$ in $b$ with $s<t$ and $p_{s}>p_{t}$, then there is some $r<s$ with $p_{r}>p_{s}$, since $p_{s}$ is not in $a$. But then $p_{r} p_{s} p_{t}$ forms a 321 pattern in $p$.

Let $\omega \in \widetilde{S}_{n}$ and suppose that for some $1 \leqslant \alpha<\beta \leqslant n$, we have

$$
\left\lfloor\frac{\left|\omega_{\beta}-\omega_{\alpha}\right|}{n}\right\rfloor>m^{\ell+1}+1 .
$$

If $\omega_{\alpha}<\omega_{\beta}$, set $\omega_{\alpha}^{\prime}=\omega_{\beta}$ and $\omega_{\beta}^{\prime}=\omega_{\alpha}+n$. Then we will have $\omega_{\alpha}^{\prime}>\omega_{\beta}^{\prime}$ and

$$
\left\lfloor\frac{\left|\omega_{\beta}^{\prime}-\omega_{\alpha}^{\prime}\right|}{n}\right\rfloor>m^{\ell+1} .
$$

So in what follows we will assume $\omega_{\alpha}>\omega_{\beta}$ and

$$
\left\lfloor\frac{\left|\omega_{\beta}-\omega_{\alpha}\right|}{n}\right\rfloor>m^{\ell+1} .
$$

We can now construct the occurrence of $p$ in $\omega$. Our iterative algorithm will complete in $\ell$ steps, where $\ell$ is the length of the subsequence $a$ described above. We will be using 


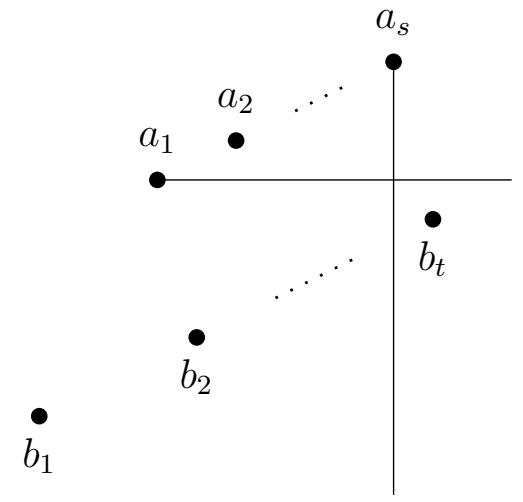

Figure 2: First place all values of $p$ to the left of $b_{t}$.

translates $\omega_{\alpha+k n}$ to place the terms of $p$ in the $a$ sequence and translates $\omega_{\beta+k n}$ to place the terms of $p$ in the $b$ sequence.

Since $p$ is not decomposable, $a_{1} \neq 1$. Hence there is some $t$ such that $b_{t}=a_{1}-1$. Suppose $b_{t}=p_{i}$. Let $s$ be the largest index such that $a_{s}$ lies to the left of $b_{t}$ in $p$. Note that $1<s<m$ or else $p$ is decomposable. Let $y$ be the largest integer such that $\omega_{\beta+y n}<\omega_{\alpha}$ and let $z=\lfloor\underline{s}\rfloor$. Since $\omega_{\alpha}-\omega_{\beta}>n m^{\ell+1}$, we have $y>m^{\ell+1}$ and hence $z>m^{\ell}$. For each $1 \leqslant k \leqslant s$, use $\omega_{\alpha+(k-1) z n}$ to place $a_{k}$ in $\omega$. Then if $\omega_{u}$ corresponds to $a_{k}$ and $\omega_{v}$ corresponds to $a_{k+1}$, we will have

$$
\left|\omega_{u}-\omega_{v}\right|=|u-v|=n z>n m^{\ell} .
$$

Finally, use translates of $\omega_{\beta}$ to place $b_{1}, \ldots, b_{t}$ in $\omega$ in such a way that $b_{t}$ is placed at $\omega_{\beta+y n}$ and for any $1 \leqslant x<t$, if $b_{x}$ lies between $a_{k}$ and $a_{k+1}$ in $p$, then $b_{x}$ is placed at a translate of $\omega_{\beta}$ between $\omega_{\alpha+(k-1) z n}$ and $\omega_{\alpha+k z n}$. By (8) there are at least $m^{\ell}$ translates of $\omega_{\beta}$ in this interval, so there is enough space to place all of the $b_{x}$ 's that lie between $a_{k}$ and $a_{k+1}$ using translates of $\omega_{\beta}$. Thus after the first iteration we have placed $p_{1} \cdots p_{i}$ in $\omega$.

Now suppose we have placed every term in the $a$ sequence up to $a_{r}$ for some $1<r<\ell$. If we have placed $a_{r}$, then we have also placed some additional terms from the $b$ sequence. Again, fix $t$ so that $b_{t}$ is the largest element in $p$ to the right of $a_{r}$ satisfying $b_{t}<a_{r}$. We may assume such a $b_{t}$ exists, or else $p$ is decomposable. If $b_{t}=p_{i}$, then we have actually placed $p_{1} \cdots p_{i}$. Moreover, suppose that the terms from the $a$ sequence among $p_{1} \cdots p_{i}$ have been placed so that if $\omega_{u}$ corresponds to $a_{k}$ and $\omega_{v}$ corresponds to $a_{k+1}$ for some $1 \leqslant k \leqslant r$, then

$$
\left|\omega_{u}-\omega_{v}\right|=|u-v|>n m^{\ell-r+1} .
$$

Note we must have also already placed $a_{r+1}$, or else $a_{r+1}=p_{i+1}$ and hence $p$ is decomposable.

We will now show how to place all terms in $p$ from the $b$ sequence whose values are between $a_{r}$ and $a_{r+1}$, thus completing the $(r+1)^{\text {st }}$ step of our algorithm. Note that in the process of placing these terms, we will also possibly be placing some additional terms from the $a$ sequence. Let $\omega_{u}$ correspond to $a_{r}$ and $\omega_{v}$ correspond to $a_{r+1}$. Then we have at least 


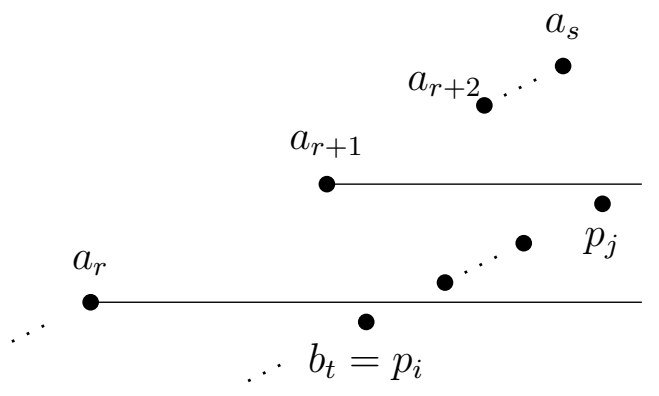

Figure 3: The $(r+1)^{\text {st }}$ iteration will place all elements of $p$ between $p_{i+1}$ and $p_{j}$.

$m^{\ell-r+1}$ translates of $\omega_{\alpha}$ and $\omega_{\beta}$ falling between $\omega_{u}$ and $\omega_{v}$. So if $p_{j}$ is the largest entry of $p$ to the left of $a_{r+1}$ satisfying $p_{j}<a_{r+1}$, as in the first step of our algorithm, we may place $p_{i+1}, \ldots, p_{j}$ in such a way that any of the terms corresponding to the subsequence $a$ are placed at least $m^{\ell-r}$ translates apart.

Iterating this algorithm $\ell$ times will place all of $p$ in $\omega$. Hence if $\omega$ is to avoid $p$, then we must have

$$
\left\lfloor\frac{\left|\omega_{\beta}-\omega_{\alpha}\right|}{n}\right\rfloor \leqslant m^{\ell+1}+1 \text { for all } 1 \leqslant \alpha<\beta \leqslant n .
$$

Since $\operatorname{inv}\left(\omega_{1}, \ldots, \omega_{n}\right) \leqslant\left(\begin{array}{l}n \\ 2\end{array}\right)$, we conclude by $(6)$ that

$$
\ell(\omega) \leqslant\left(\begin{array}{l}
n \\
2
\end{array}\right)+\left(m^{\ell+1}+1\right)\left(\begin{array}{l}
n \\
2
\end{array}\right)=\left(m^{\ell+1}+2\right)\left(\begin{array}{l}
n \\
2
\end{array}\right) .
$$

In other words, if $\ell(\omega)>\left(m^{\ell+1}+2\right)\left(\begin{array}{l}n \\ 2\end{array}\right)$, then $\omega$ will contain $p$.

For any $k$, the set of all affine permutations in $\widetilde{S}_{n}$ of length at most $k$ is finite. Hence there can be only finitely many elements in $\widetilde{S}_{n}$ that avoid $p$.

Note that in general, the length bound $\ell(\omega) \leqslant\left(m^{\ell+1}+2\right)\left(\begin{array}{l}n \\ 2\end{array}\right)$ is much larger than needed. For the proof of Theorem 1 though, any upper bound on $\ell(\omega)$ will suffice. Given a specific pattern $p$, we can tighten the bounds in the above algorithm, and thus obtain better upper bounds on the maximal length for pattern avoidance.

For example, let $p=3412 \in S_{4}$. By (10), if $\omega \in \widetilde{S}_{n}$ avoids $p$, then $\ell(\omega) \leqslant 66\left(\begin{array}{l}n \\ 2\end{array}\right)$. Here the algorithm is completed on the first iteration and we can actually prove a tighter bound $\ell(\omega) \leqslant 3\left(\begin{array}{l}n \\ 2\end{array}\right)$ for this particular pattern.

\section{Generating Functions for Patterns in $S_{3}$}

Let $f_{n}^{p}$ and $f^{p}(t)$ be as in (1) and (2) in Section 1 . Then by Theorem 1 we have $f_{n}^{321}=\infty$ for all $n$. However, for all of the other patterns $p \in S_{3}$ we can still compute $f^{p}(t)$. 
Theorem 6. Let $f^{p}(t)$ be as above. Then

$$
\begin{gathered}
f^{123}(t)=0 \\
f^{132}(t)=f^{213}(t)=\sum_{n=2}^{\infty} t^{n} \\
f^{231}(t)=f^{312}(t)=\sum_{n=2}^{\infty}\left(\begin{array}{c}
2 n-1 \\
n
\end{array}\right) t^{n} .
\end{gathered}
$$

To make the proof easier, we first study a few operations on $\widetilde{S}_{n}$ that interact with pattern avoidance in a predictable way.

Lemma 7. Let $\omega \in \widetilde{S}_{n}$ and $p \in S_{m}$. Then $\omega$ avoids $p$ if and only if $\omega^{-1}$ avoids $p^{-1}$.

Proof. The proof is the same as the one for non-affine permutations given in [West, 1990, Lemma 1.2.4]. Suppose $\omega$ contains $p$, so that $\omega_{i_{1}} \omega_{i_{2}} \cdots \omega_{i_{m}}$ is an occurrence of $p$ in $\omega$. Let $j_{k}=\omega_{i_{k}}$ for $1 \leqslant k \leqslant m$. Then $\omega_{j_{1}}^{-1} \cdots \omega_{j_{m}}^{-1}$ will give an occurrence of $p^{-1}$ in $\omega^{-1}$.

Now define a map $\sigma_{r}: \widetilde{S}_{n} \rightarrow \widetilde{S}_{n}$ by setting

$$
\sigma_{r}(\omega)_{i}= \begin{cases}\omega_{i-1}+1, & \text { if } 2 \leqslant i \leqslant n \\ \omega_{n}-n+1, & \text { if } i=1\end{cases}
$$

This has the effect of shifting the base window of $\omega$ one space to the right, while preserving the relative order of the elements. The affine inversion table of $\sigma_{r}(\omega)$ is a barrel shift of the affine inversion table of $\omega$ one space to the right. Similarly, define $\sigma_{\ell}=\sigma_{r}^{-1}$, which will perform a barrel shift one space to the left. Thus $\sigma_{r}$ is the length-preserving automorphism of $\widetilde{S}_{n}$ of order $n$ obtained by rotating the Coxeter graph one space clockwise.

For example, if $\omega=[5,-4,6,3] \in \widetilde{S}_{4}$, which has affine inversion table $(4,0,3,1)$, then $\sigma_{r}(\omega)=[0,6,-3,7]$, which has affine inversion table $(1,4,0,3)$.

Lemma 8. Let $\omega \in \widetilde{S}_{n}$ and $p \in S_{m}$. The following are equivalent.

1. $\omega$ avoids $p$.

2. $\sigma_{r}(\omega)$ avoids $p$.

3. $\sigma_{\ell}(\omega)$ avoids $p$.

Proof. The relative order of elements in $\omega$ is unchanged after applying $\sigma_{r}$ or $\sigma_{\ell}$. Hence if $\omega_{i_{1}} \cdots \omega_{i_{m}}$ is an occurrence of $p$ in $\omega$, then $\omega_{i_{1}+1} \cdots \omega_{i_{m}+1}$ is an occurrence of $p$ in $\sigma_{r}(\omega)$ and $\omega_{i_{1}-1} \cdots \omega_{i_{m}-1}$ is an occurrence of $p$ in $\sigma_{\ell}(\omega)$.

We are now ready to enumerate the affine permutations that avoid a given pattern in $S_{3}$ 
Proof of Theorem 6. For any $\omega \in \widetilde{S}_{n}$, the entries $\omega_{1} \omega_{1+n} \omega_{1+2 n}$ are always an occurrence of 123 in $\omega$. Hence $f_{n}^{123}=0$ for all $n$. If $\omega$ has a descent at $\omega_{i}$ so that $\omega_{i}>\omega_{i+1}$, then there is some translate $i-s n$ such that $\omega_{i-s n}<\omega_{i+1}$. Hence $\omega_{i-s n} \omega_{i} \omega_{i+1}$ is an occurrence of 132 in $\omega$. Also, $\omega_{i+n}>\omega_{i+1}$ so that $\omega_{i} \omega_{i+1} \omega_{i+n}$ is an occurrence of 213 in $\omega$. Thus the only affine permutation that can avoid 132 or 213 is the identity. Hence $f_{n}^{132}=f_{n}^{213}=1$.

By Lemma 7 we have $f_{n}^{231}=f_{n}^{312}$. Thus it remains to compute $f_{n}^{231}$. So suppose $\omega$ avoids 231. We first show $\omega$ is in a proper parabolic subgroup that depends on the position and value of the maximal element of the base window.

Let $\alpha$ be the index such that $\omega_{\alpha}=\max \left\{\omega_{1}, \ldots, \omega_{n}\right\}$. First suppose $\omega_{\alpha}>n+\alpha-1$. Shift $\omega$ to the left $\alpha-1$ times, setting $\nu=\sigma_{\ell}^{\alpha-1}(\omega)$. Then $\nu_{1}=\omega_{\alpha}-\alpha+1>n$. Since $\nu$ must satisfy (5), there must exist some $1<j \leqslant n$ with $\nu_{j} \leqslant 0$. Then $\nu_{1-n} \nu_{1} \nu_{j}$ is an occurrence of 231 in $\nu$. By Lemma 8, $\omega$ contains 231, which is a contradiction. So we must have $n \leqslant \omega_{\alpha} \leqslant n+\alpha-1$.

Now let $u=\sigma_{\ell}^{\omega_{\alpha}-n}(\omega)$. Set $i=\alpha-\omega_{\alpha}+n$ so that $u_{i}=n$. If $\left\{u_{1}, \ldots, u_{n}\right\} \neq[n]$, then since $u$ must satisfy (5), there is some $1 \leqslant j, k \leqslant n$ such that $u_{j}<0$ and $u_{k}>n$. Since $\omega_{\alpha}$ was chosen to be maximal, we must have $i<k$. Then $u_{i} u_{k} u_{j+n}$ will give an occurrence of 231 in $u$ and hence also in $\omega$ by Lemma 8, giving a contradiction. Hence $u \in S_{n} \subset \widetilde{S}_{n}$.

Let $C_{n}=\frac{1}{n+1}\left(\begin{array}{c}2 n \\ n\end{array}\right)$ be the $n^{\text {th }}$ Catalan number. Recall from Knuth [1973] that there are $C_{n}$ 231-avoiding permutations in $S_{n}$. Again, suppose $\omega_{\alpha}=\max \left\{\omega_{1}, \ldots, \omega_{n}\right\}$ and $\omega_{\alpha}=n+\alpha-i$, for some $1 \leqslant i \leqslant \alpha$. Then $u=\sigma_{\ell}^{\omega_{\alpha}-n}(\omega)$ is an element in $S_{n}$ with $u_{i}=n$. Furthermore, we have $u_{h}<u_{j}$ for every pair $h<i<j$. There are $C_{i-1} C_{n-i}$ such permutations. Summing over all possible values of $i$ gives

$$
\sum_{i=1}^{\alpha} C_{i-1} C_{n-i}=\sum_{i=0}^{\alpha-1} C_{i} C_{n-1-i}
$$

many 231-avoiding affine permutations whose maximal value in the base window occurs at index $\alpha$. Summing over all $1 \leqslant \alpha \leqslant n$ then gives

$$
f_{n}^{231} \leqslant \sum_{\alpha=1}^{n}\left(\sum_{i=0}^{\alpha-1} C_{i} C_{n-1-i}\right) .
$$

Using the defining recurrence,

$$
C_{n}=\sum_{i=0}^{n-1} C_{i} C_{n-1-i}
$$

for the Catalan numbers, (14) simplifies to

$$
f_{n}^{231} \leqslant \frac{(n+1)}{2} C_{n}=\left(\begin{array}{c}
2 n-1 \\
n
\end{array}\right)
$$

Conversely, if $u \in S_{n} \subset \widetilde{S}_{n}$ is a 231-avoiding permutation with $u_{i}=n$, then $\sigma_{r}^{j}(u)$ will be a 231-avoiding affine permutation for any $0 \leqslant j \leqslant n-i$. Thus we actually have equality in (16), completing the proof. 


\section{Generating Functions for Patterns in $S_{4}$}

We now look at pattern avoidance for patterns in $S_{4}$. There are 24 patterns to consider, although for all but three patterns, $f^{p}(t)$ is easy to compute. First let

$$
P=\{1432,2431,3214,3241,3421,4132,4213,4231,4312,4321\}
$$

By Theorem 1 , if $p \in P$, then $f_{n}^{p}=\infty$, so $f^{p}(t)$ is not defined.

Theorem 9. We have

$$
\begin{gathered}
f^{1234}(t)=0 \\
f^{1243}(t)=f^{1324}(t)=f^{2134}(t)=f^{2143}(t)=\sum_{n=2}^{\infty} t^{n} \\
f^{1342}(t)=f^{1423}(t)=f^{2314}(t)=f^{3124}(t)=\sum_{n=2}^{\infty}\left(\begin{array}{c}
2 n-1 \\
n
\end{array}\right) t^{n} .
\end{gathered}
$$

Proof. As in Theorem 6 there are no affine permutations avoiding 1234, and only the identity permutation avoids $1243,1324,2134$, or 2143 . If $\omega_{i_{1}} \omega_{i_{2}} \omega_{i_{3}}$ is an occurrence of 231 in $\omega$, then there is some translate $i_{1}-s n$ such that $\omega_{i_{1}-s n}<\omega_{i_{3}}$. Hence $\omega_{i_{1}-s n} \omega_{i_{1}} \omega_{i_{2}} \omega_{i_{3}}$ is an occurrence of 1342 in $\omega$. Conversely, if $\omega$ avoids 231, then it must also avoid any pattern containing 231, namely 1342. This shows $f_{n}^{1342}=f_{n}^{231}$. Similarly, we also have $f_{n}^{1423}=f_{n}^{2314}=f_{n}^{3124}=f_{n}^{231}$.

Based on some initial calculations, we also have the following conjectures for the remaining patterns in $S_{4}$.

Conjecture 1. The following equalities hold:

$$
\begin{aligned}
& f_{n}^{3142}=f_{n}^{2413}=\sum_{k=0}^{n-1} \frac{(n-k)}{n}\left(\begin{array}{c}
n-1+k \\
k
\end{array}\right) 2^{k} \\
& f_{n}^{3412}=f_{n}^{4123}=f_{n}^{2341}=\frac{1}{3} \sum_{k=0}^{n}\left(\begin{array}{l}
n \\
k
\end{array}\right)^{2}\left(\begin{array}{c}
2 k \\
k
\end{array}\right) .
\end{aligned}
$$

Note that (20) is sequence A064062 and (21) is sequence A087457 in Sloane [2009]. It is also worth comparing (21) to the number of 3412-avoiding, non-affine permutations given in [Gessel, 1990, §7] as

$$
u_{3}(n)=2 \sum_{k=0}^{n}\left(\begin{array}{l}
n \\
k
\end{array}\right)^{2}\left(\begin{array}{c}
2 k \\
k
\end{array}\right) \frac{3 k^{2}+2 k+1-n-2 k n}{(k+1)^{2}(k+2)(n-k+1)} .
$$




\section{Acknowledgements}

The author would like to thank Brant Jones for inspiring him to ask this question and Sara Billey for all of her guidance and helpful conversations. He is also indebted to Neil Sloane and his team, whose encyclopedia of integer sequences was invaluable for forming the conjectures in this paper. Thanks to the anonymous referee, particularly for the recommendation on simplifying the proof of Theorem 1.

\section{References}

S. Billey and S. A. Mitchell. Affine partitions and affine Grassmannians. Electron. J. Combin., 16(2):Research Paper 18, 45 pp. (electronic), 2009.

S. Billey, W. Jockusch, and R. P. Stanley. Some combinatorial properties of Schubert polynomials. J. Algebraic Combin., 2(4):345-374, 1993. ISSN 0925-9899.

A. Björner and F. Brenti. Combinatorics of Coxeter groups, volume 231 of Graduate Texts in Mathematics. Springer, New York, 2005. ISBN 978-3540-442387; 3-540-44238-3.

A. Björner and F. Brenti. Affine permutations of type A. Electron. J. Combin., 3(2): Research Paper 18, approx. 35 pp. (electronic), 1996. ISSN 1077-8926. The Foata Festschrift.

I. M. Gessel. Symmetric functions and P-recursiveness. J. Combin. Theory Ser. A, 53(2):257-285, 1990. ISSN 0097-3165. doi: 10.1016/0097-3165(90)90060-A. URL http://dx.doi.org/10.1016/0097-3165(90)90060-A.

R. M. Green. On 321-avoiding permutations in affine Weyl groups. J. Algebraic Combin., 15(3):241-252, 2002. ISSN 0925-9899.

C. Hanusa and B. Jones. The enumeration of fully commutative affine permutations. preprint, math.CO/0907.0709v1, July 2009.

J. E. Humphreys. Reflection groups and Coxeter groups, volume 29 of Cambridge Studies in Advanced Mathematics. Cambridge University Press, Cambridge, 1990. ISBN 0-521$37510-\mathrm{X}$.

D. E. Knuth. The art of computer programming. Volume 3. Addison-Wesley Publishing Co., Reading, Mass.-London-Don Mills, Ont., 1973. Sorting and searching, AddisonWesley Series in Computer Science and Information Processing.

V. Lakshmibai and B. Sandhya. Criterion for smoothness of Schubert varieties in SL $(n) / B$. Proc. Indian Acad. Sci. Math. Sci., 100(1):45-52, 1990. ISSN 0253-4142.

G. Lusztig. Some examples of square integrable representations of semisimple $p$-adic groups. Trans. Amer. Math. Soc., 277(2):623-653, 1983. ISSN 0002-9947. 
A. Marcus and G. Tardos. Excluded permutation matrices and the Stanley-Wilf conjecture. J. Combin. Theory Ser. A, 107(1):153-160, 2004. ISSN 0097-3165.

J. Y. Shi. The Kazhdan-Lusztig cells in certain affine Weyl groups, volume 1179 of Lecture Notes in Mathematics. Springer-Verlag, Berlin, 1986. ISBN 3-540-16439-1.

N. J. A. Sloane. Online encyclopedia of integer sequences. http://www.research.att.com/ njas/sequences/, 2009.

J. R. Stembridge. On the fully commutative elements of Coxeter groups. J. Algebraic Combin., 5(4):353-385, 1996. ISSN 0925-9899.

B. E. Tenner. Pattern avoidance and the Bruhat order. J. Combin. Theory Ser. A, 114 (5):888-905, 2007. ISSN 0097-3165.

B. E. Tenner. Database of permutation pattern avoidance. http://math.depaul.edu/ bridget/patterns.html, 2009.

J. West. Permutations with forbidden sequences; and, stack-sortable permutations. PhD thesis, Massachusetts Institute of Technology, September 1990. 